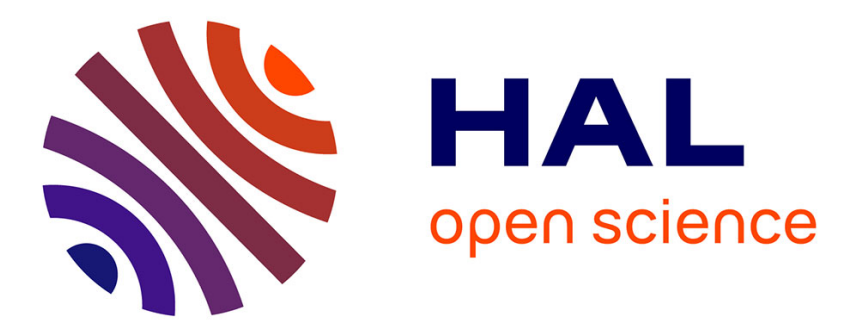

\title{
DETERMINATION OF NUCLEAR GROUND STATE PROPERTIES FAR FROM STABILITY BY OPTICAL PUMPING
}

\author{
E. Otten
}

\section{- To cite this version:}

E. Otten. DETERMINATION OF NUCLEAR GROUND STATE PROPERTIES FAR FROM STABILITY BY OPTICAL PUMPING. Journal de Physique Colloques, 1973, 34 (C4), pp.C4-63-C4-68. 10.1051/jphyscol:1973408 . jpa-00215283

\section{HAL Id: jpa-00215283 https://hal.science/jpa-00215283}

Submitted on 1 Jan 1973

HAL is a multi-disciplinary open access archive for the deposit and dissemination of scientific research documents, whether they are published or not. The documents may come from teaching and research institutions in France or abroad, or from public or private research centers.
L'archive ouverte pluridisciplinaire $\mathbf{H A L}$, est destinée au dépôt et à la diffusion de documents scientifiques de niveau recherche, publiés ou non, émanant des établissements d'enseignement et de recherche français ou étrangers, des laboratoires publics ou privés. 


\title{
DETERMINATION OF NUCLEAR GROUND STATE PROPERTIES FAR FROM STABILITY BY OPTICAL PUMPING
}

\author{
E. W. OTTEN \\ Institut für Physik, Johannes Gutenberg-Universität, Mainz, Germany
}

\begin{abstract}
Résumé. - L'étude par pompage optique d'isotopes provenant d'une séparation de masse en ligne est discutée. Cette possibilité de détermination de spin et moments nucléaires ainsi que de la variation du volume de charge est décrite pour l'exemple de la chaîne isotopique ${ }^{181} \mathrm{Hg}-{ }^{205} \mathrm{Hg}$. La variation du volume de charge nucléaire $\left(\delta\left\langle r^{2}\right\rangle\right)$ sur cette chaîne est discutée, en particulier son changement abrupt pour les isotopes $\mathrm{Hg}$ les plus légers, ce qui indique une transition de phase dans la structure nucléaire.
\end{abstract}

Abstract. - The possibilities of determining nuclear spin, moments and variation in charge volume by optical pumping of on line masseparated isotopes are described for the example of the isotopic chain $181 \mathrm{Hg}^{205} \mathrm{Hg}$. The variation in nuclear charge volume $\left.\left(\delta<r^{2}\right\rangle\right)$ along this chain and in particular its abrupt change for the lightest $\mathrm{Hg}$-isotopes, indicating a phase transition in nuclear structure, are discussed.

1. Introduction. - This talk is based on experiments [1], [2], [3] performed recently by a visiting team $\left(^{*}\right)$ at the ISOLDE facility at CERN with the members : J. Bonn, G. Huber, H.-J. Kluge, U. Köpf, L. Kugler, J. Rodriguez, and E. W. Otten.

The investigation of the hyperfine structure (hfs) and isotopic shift (IS) of optical spectra allows the determination of the basic nuclear ground state properties : spin $(\mathrm{I})$, magnetic moment $\left(\mu_{\mathrm{I}}\right)$, electric quadrupole moment $\left(Q_{1}\right)$, and change of the mean squared radius of the nuclear charge distribution between different isotopes $\left(\delta<r^{2}>\right)$. The subject has therefore widely been explored in the past for stable and long lived isotopes [4], [5] and contributed substantially to the systematic topography of nuclear structure in the valley of stability which could be established within the last two decades. Still basical and quantitative questions are open in understanding nuclear matter. In this situation it makes from first principles is missing. In this situation it makes sense to enlarge the scope of investigations principally. In particular we should be in a position to choose and change systematically the parameters $N$ and $Z$ of a nucleus independently and free from the boundary condition of being close to the stable valley. With other words, a systematic investigation of nuclear properties along lines of constant $Z$ or $N$ across the full array of nuclei which we can reach in our days, would give us a much wider and more reliable view on the topography of nuclear structure

$\left.{ }^{*}\right)$ In the course of the experiments the team changed its home university from Heidelberg to Mainz. than just the cross section through the stable valley. Take for example the question of superheavy nuclei. Theoreticians have made detailed predictions about their properties by extrapolating from the knowledge on known nuclei. One should prove whether these theoretical methods lead to reliable statements on (lighter) nuclei far from stability, where experimental material has been made available already now.

2. The ISOLDE facility at CERN. - The ISOLDE at CERN, a collaboration of Danish, French, German, Norwegian and Swedish scientists has been a very sucessfull outrider in the research on nuclei far from stability. The ISOLDE facility [6] is a mass separator set up on line with a hot target (molten metal usually) which is irradiated by $600 \mathrm{MeV}$ protons. Spallation leeds to a great number of daughter nuclei centered on the neutron deficient side of the nuclear chart. They have to be seperated regarding $Z$ and $N$ before investigation. The separation of the element is chosen by selective evaporation from the hot target. Taking a molten $\mathrm{Pb}$ target for instance, $\mathrm{Hg}$ is the only volatile daughter element in the neighbourhood. The $\mathrm{Hg}$ vapor enters the ion source of a mass separator delivering a chain of about thirty isotopes with a yield reaching $10^{8}$ nuclei per mass number and second (see Fig. 1). The facility can provide seperated isotopes from more than thirty elements. Because of the great number of isotopes the facility usually feeds several experiments simultaneously, most of them attacking their problem by the various tools of $\alpha, \beta$, $\gamma$ spectroscopy. 


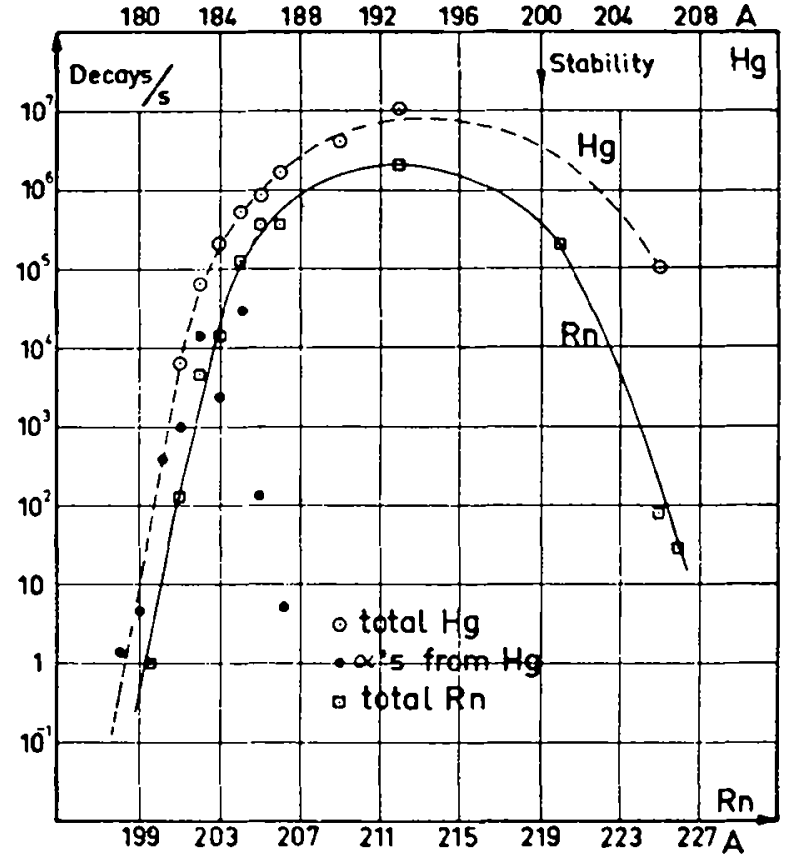

Fig. 1. - Production rate of mercury isotopes versus mass number.

3. The optical pumping on line method. - Hfs investigations of isotopes far from stability (or generally of : short lived isotopes) by optical or rf-spectroscopic methods encounter several difficulties. (i) a technical one : the methods have to be adapted to on line conditions. The times for preparation and investigation of the samples shrink down to seconds. (ii) a physical one : The number of atoms produced in saturation is generally too small to apply standard signal detection methods. The sensitivity has to be increased therefore by using the nuclear radiation instead of the photons or rf quanta. This problem has been solved in various elegant ways; for applications in connection with on line separators the atomic beam resonance and the optical pumping method are among the most promising ones (for a discussion see ref. [7], [8]).

The principle idea of Kastler' $s$ method of optical pumping (OP) is to polarize the total angular momentum $F=I+J$ of the atomic ground state by irradiation of circularly polarized resonance radiation, thus transfering the polarized angular momentum of the photons by the process of resonance absorption and reemission (pumping) to the atoms [9], [10]. The detailed mechanism of OP is shown in figure 2 for the $\mathrm{Hg}$ atom, the case of our interest where a nuclear spin $I=1 / 2$ has been assumed for simplicity. The diamagnetic ground state ${ }^{1} \mathrm{~S}_{0}$ has total angular momentum $F=I=1 / 2$ because of $J=0$. It splits into two nuclear Zeeman levels $m_{\mathrm{I}}=+1 / 2$ and $-1 / 2$. In the excited ${ }^{3} P_{1}$ state one obtains two hyperfine components with $F=1 / 2,3 / 2$ which split into two and four sublevels, respectively. Let us assume that the optical excitation via the resonance line excites only one of the upper $F$ levels, either the $F=1 / 2$ or the $F=3 / 2$ level. In $\mathrm{Hg}$ this is possible in general, since the hfs splitting is large as compared to the

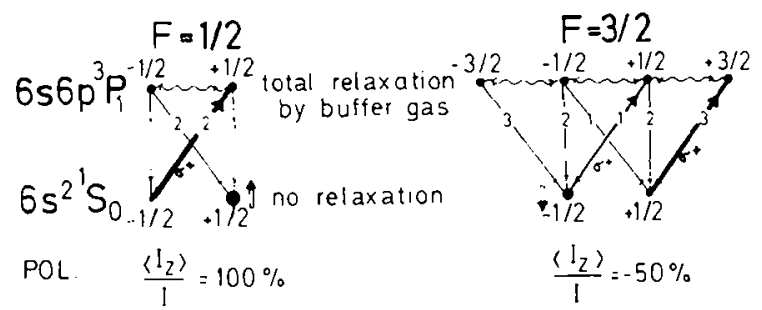

Frg. 2. - OP cycle for a mercury isotope with $I=1 / 2$. The thick arrows characterize the $\Delta m_{F}=+1$ absorptions. Since the presence of a buffer gas causes total relaxation in the nonspherical $3 \mathbf{P}_{1}$ state (wavy line) the spontaneous emissions take place via all indicated transitions. The numbers in between are relative absorption and emission rates starting from an unpolarized atom. The obtained nuclear polarization in the limit of saturation of $\mathrm{OP}$ is given for pumping via the $F=1 / 2$ and $3 / 2$ state, respectively.

Doppler width of the spectral line (compare also figure 4). It is easily shown from the particulars of figure 2 that $O P$ will leed in saturation (neglecting relaxations in the groundstate) to population numbers of the $\left|m_{\mathrm{I}}\right\rangle$ substates being proportional to the inverse of the relative absorption probabilities. Thus $\left\langle I_{z}\right\rangle I I=100 \%$ and $-50 \%$ are obtained for $F=1 / 2$ and $F=3 / 2$ pumping resp. Note the role of the buffer gas (noblegas). It causes a complete relaxation of the non spherical excited state within its lifetime of $10^{-7} \mathrm{~s}$, but leaves the nucleus in the surrounding of the spherical electronic configuration of the atomic groundstate polarized for seconds. Its main purpose is to prevent too fast relaxations at the paramagnetic walls of the resonance vessel.

For an atomic vapor of reasonable optical thickness the OP effect can be monitored easily by a change in the absorption of the pumping light, since the levels have different relative absorption strength. In the case of a small number of radioactive atoms this may not be detectable, but we have the chance to monitor the polarization (or alignment if $I>1 / 2$ ) by the asymmetry of $\beta$-decay :

$$
W_{\beta}(\vartheta)=1+\frac{v}{c} \frac{<I_{z}}{I} \geq A \cos \vartheta
$$

or the $\gamma$-decay anisotropy :

$$
W_{, \gamma}(\vartheta)=\sum_{k \text { even }} B_{k} F_{k} P_{k}(\cos \vartheta)
$$

$\left(P_{k}\right.$ are the Legendre polynomials; the coefficients $F_{k}$ are determined by the degree of nuclear orientation, $B_{k}$ by the $\gamma$-decay mode, both defined in the literature on angular correlations). 
OP experiments based on the $\beta$-detection principle were first performed on some shortlived mirror nuclei in order to determine their moment [11], [12], [13], [14]. A $\gamma$ anisotropy after optical pumping was first observed for the case of ${ }^{203} \mathrm{Hg}$ [15]. The experiments described below are an extension of the methods to isotopes far from stability, delivered by on line mass separators.

The on-line OP set-up is shown in figure 3. The mercury ion beam coming from the separator is stopped on one of the two molybdenum foils. After a collection time of about onc half-life, foil $\mathrm{I}$ is turned, foil II now collects the ion beam and foil $I$ is heated out at temperatures of above $1000^{\circ} \mathrm{C}$. Together with a stream of helium buffer gas the mercury atoms enter the quartz vessel. For OP the resonance cell is illuminated by the $\left(6 \mathrm{~s} 6 \mathrm{p}{ }^{3} \mathrm{P}_{1}-6 \mathrm{~s}^{2}{ }^{1} \mathrm{~S}_{0}, \lambda=2537 \AA\right)$ radiation of a mercury microwave lamp. The circular polarization of the pumping light is periodically alternated by inserting a $\lambda / 2$ birefringent quartz plate. Thus, the sign of the nuclear polarization is reversed and any instrumental asymmetry avoided. $\beta$-radiation

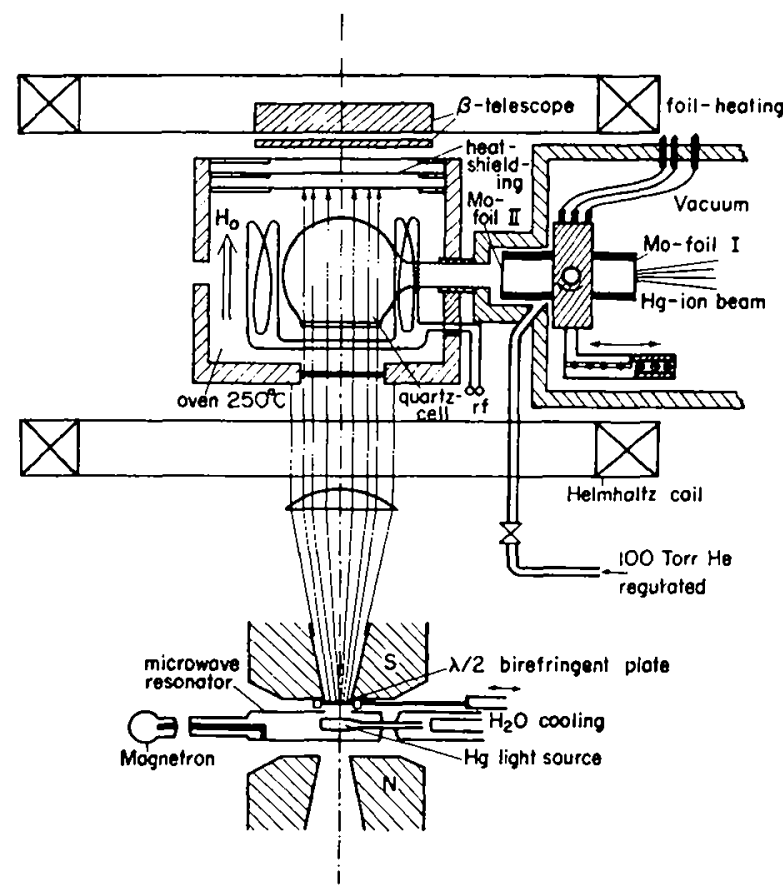

Fig. 3. - Principle of the apparatus for on-line investigation and polarization of shortlived mercury isotopes.

is counted at $0^{\circ}, 180^{\circ}$, and $90^{\circ}$ in respect of the static magnetic field $H_{0}$ over the resonance cell. (The $\gamma$-anisotropy is counted by $\mathrm{NaI}$ crystals or GeLi diodes under $0^{\circ}$ and $90^{\circ}$ with a different polarization scheme allowing the nuclear orientation to flip between $0^{\circ}$ and $\left.90^{\circ}\right)$. The nuclear orientation can reach values different from zero only in the case of optical resonance between emission and absorption line. Since (IS) and $\mathrm{hfs}$ of this $\mathrm{Hg}$ resonance line are bigger than the doppler width of the exciting light, the lamp, containing ${ }^{204} \mathrm{Hg}$, is subjected to a magnetic field in order to match a hyperfine component of the unstable isotope by a Zeeman component of the lamp. Therefore, the decay asymmetry, plotted against the magnetic field, displays directly the hfs. Figure 4 shows the experimental $\beta$-decay asymmetry

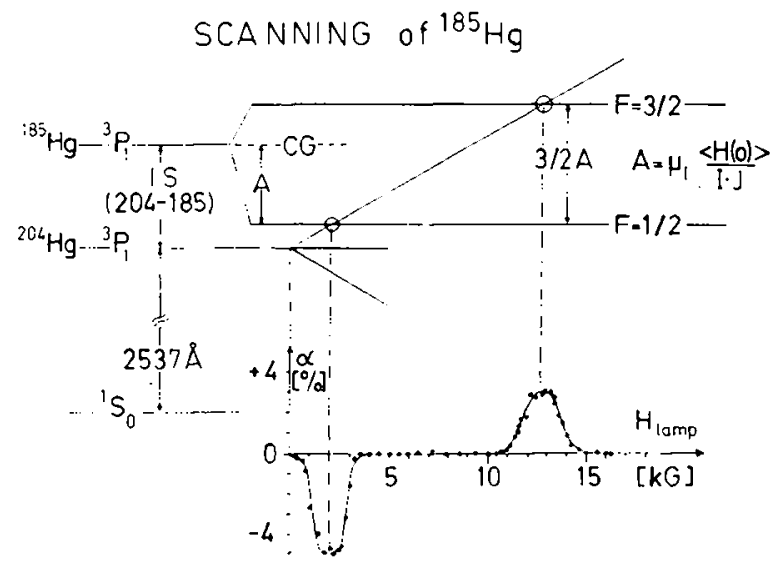

FIG. 4. - Experimental $\beta$ decay asymmetry of $185 \mathrm{Hg}$ as a function of scanning magnetic field over the light source. The lamp contains isotopically pure ${ }^{204} \mathrm{Hg}$.

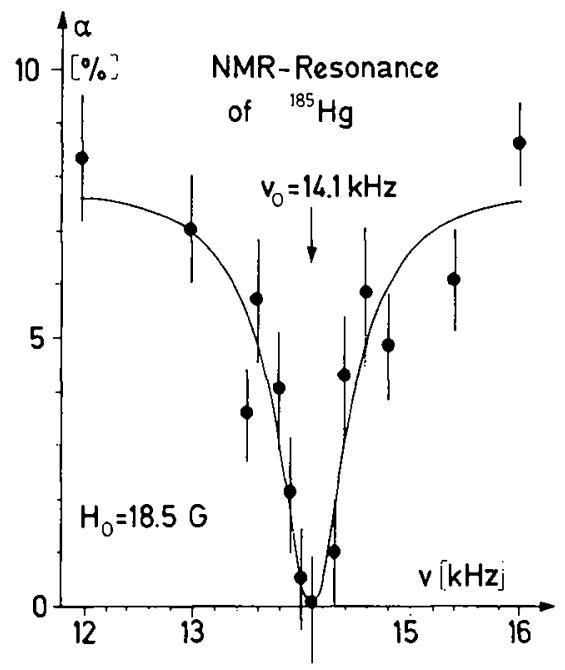

Fig. 5. - Destruction of the $\beta$ decay asymmetry of optically pumped $185 \mathrm{Hg}$ by nuclear resonance.

of ${ }^{185} \mathrm{Hg}$ as a function of the scanning magnetic field and the thereby deduced level scheme, from which I, $\mu_{\mathrm{l}}$, and IS are determined. In addition $g_{\mathrm{I}}$ can be measured directly by applying NMR to the polarized nuclei while the atoms are in their diamagnetic groundstate, thus destroying the asymmetry (Fig. 5). With a field of a few Gauss over the resonance vessel a satisfying accuracy is obtained already. In favourable cases the asymmetry can be observed directly on the chart recording of the monitor counting rate under $0^{\circ}$ (Fig. 6). 
Figure 7 gives an example for $\gamma$-decay anisotropy, observed after pumping the $I=13 / 2$ isomer of ${ }^{199} \mathrm{Hg}$. Using Ge-Li-diodes anisotropies have been

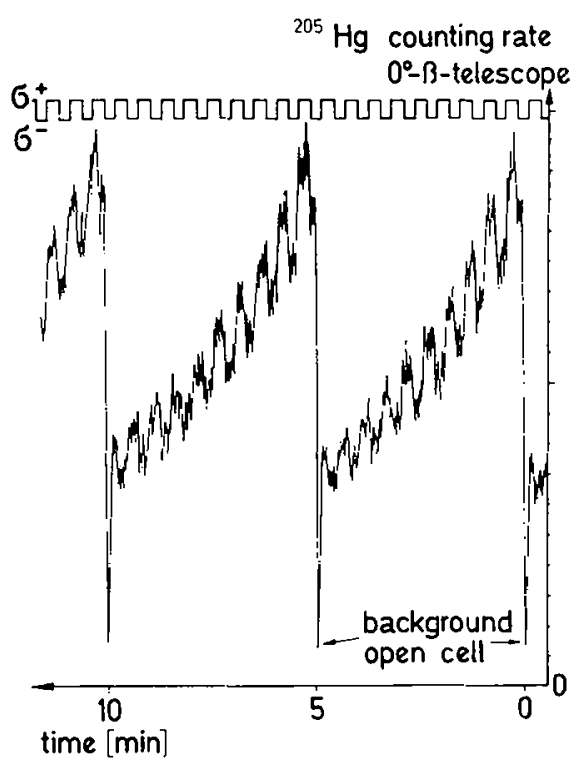

FIg. 6. - Chart recording of the monitor counting rate of $\beta$ particles emitted from polarized ${ }^{205} \mathrm{Hg}$ under $0^{\circ}$. Time runs from right to left. Two full counting periods are recorded. Inbetween the drop of activity to the background, when the old ${ }^{205} \mathrm{Hg}$ gas is pumped off, and the rise when the fresh sample streams in. Supcrimposed to the exponential decay one sees wriggles due to the change of sign of polarization.

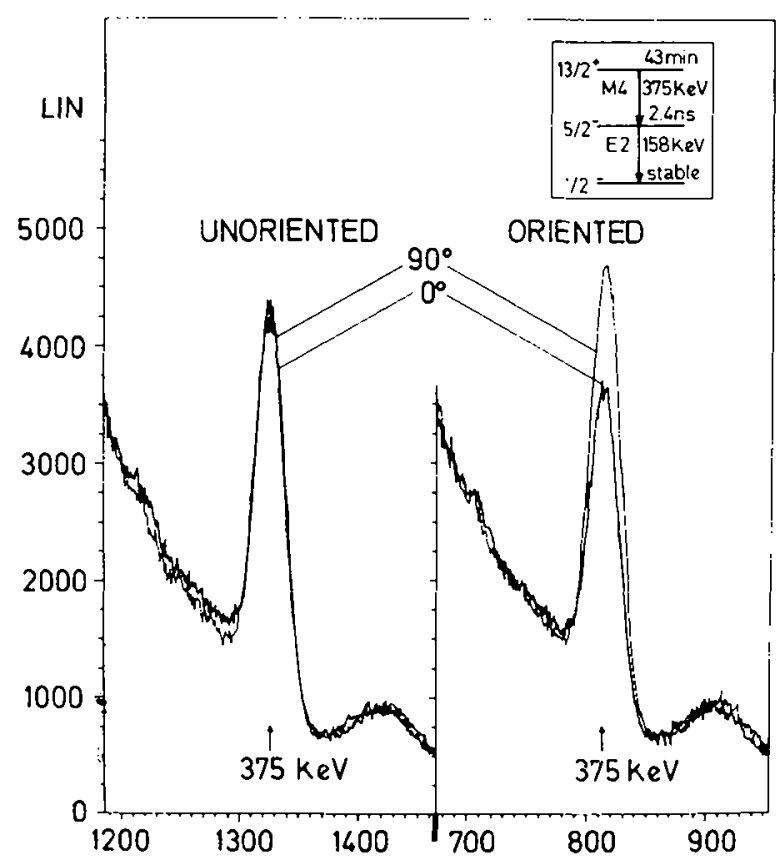

FIG. 7. - Insert : Nuclear decay scheme of the ${ }^{199} \mathrm{Hg}$ isomer. The orientation of the $13 / 2$ state was detected by the $0^{\circ}-90^{\circ}$ anisotropy of the M4 and E2 transitions. The observed effect in the M4 transition is shown by a plot of the spectrum of the $0^{\circ}$ and $90^{\circ} \mathrm{NaI}(\mathrm{Tl})$ counter in the oriented (right) and the unoriented (left) case. Normalizing the high energy background to equal counting rates, a $30 \%$ difference between $90^{\circ}$ and $0^{\circ}$ counting rate is observed in the M4 transition of the oriented nuclei. observed in most of the $\gamma$-lines of the complex decay schemes of ${ }^{193} \mathrm{Hg}$ and ${ }^{193} \mathrm{Hg}^{\mathrm{m}}$. It will be prooved whether this technique can be used for determination of spins and multipolarities in nuclear decay schemes.

4. Discussion of results. - 4.1. IsOTOPE SHIFT IN SPHERICAL Hg NUCLEI. - Figure 8 contains as function of neutron number all hfs- and IS-data, known for this spectral line spanning from ${ }^{181} \mathrm{Hg}$ to ${ }^{205} \mathrm{Hg}$. The stable isotopes are centered around ${ }^{200} \mathrm{Hg}$. The results from ${ }^{192} \mathrm{Hg}$ to ${ }^{203} \mathrm{Hg}$ including isomers, were obtained before already by standard spectroscopic methods applying off line techniques as far as radioactives were concerned [16]. They include lifetimes down to about one hour which seems to be about the lower limit for experiments of this kind [16], [17]. They established already the widest and most complete set of hfs and IS data collected for the isotopes of one element. This was a very good starting position for our experiments, the results of which are collected in table I seperately.

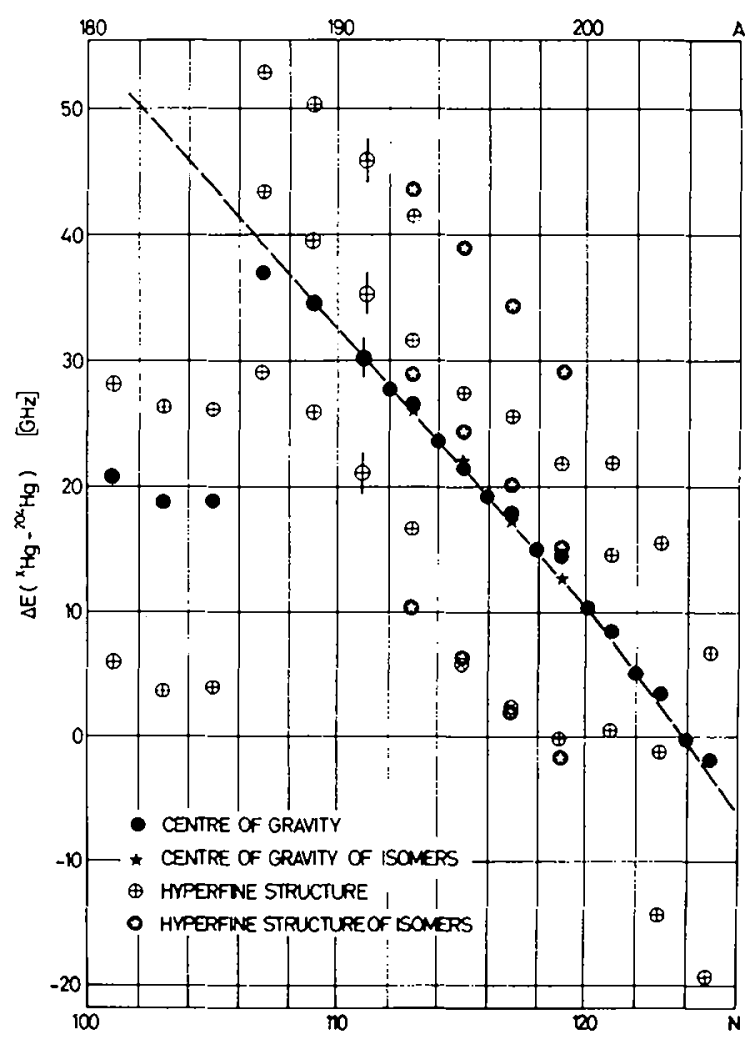

FIG. 8. - Hfs splitting and IS of $\mathrm{Hg}$ isotopes relative to ${ }^{204} \mathrm{Hg}$ in the spectral line $\left(6 \mathrm{~s}^{2} \mathrm{~S}_{0}-6 \mathrm{~s} 6 \mathrm{p}{ }^{3} \mathrm{P}_{1}, \lambda=2537 \AA\right)$. The error in the position of the hfs states (and hence of the IS) of the short lived isotopes is about the diameter of the dots.

We will restrict the discussion to the full black points of figure 8 representing the IS. For odd isotopes it is always identical with the shift of the centre of gravity of a hfs multiplet with respect to another isotope. From ${ }^{205} \mathrm{Hg}$ down to ${ }^{187} \mathrm{Hg}$ we observe a 
TABLE I

\begin{tabular}{ccl}
${ }^{x} \mathrm{Hg}$ & $T_{1 / 2}$ & \multicolumn{1}{c}{ Method } \\
$\overline{181}$ & $3.6 \mathrm{~s}$ & on line $\beta$-asy \\
183 & $8.8 \mathrm{~s}$ & on line $\beta$-asy \\
185 & $50 \mathrm{~s}$ & on line $\beta$-asy \\
187 & $2.4 \mathrm{mn}$ & on line $\beta$-asy \\
189 & $8.7 \mathrm{mn}$ & on line $\beta$-asy \\
& & \\
191 & $56 \mathrm{mn}$ & on line $\beta$-asy \\
193 & $4 \mathrm{~h}$ & on line $\gamma$-aniso \\
$193 \mathrm{~m}$ & $11 \mathrm{~h}$ & on line $\gamma$-aniso \\
$199 \mathrm{~m}$ & $43 \mathrm{mn}$ & on line $\gamma$-aniso \\
205 & $5.5 \mathrm{mn}$ & on line $\beta$-asy \\
199 & stable & standard opti- \\
201 & stable & cal methods
\end{tabular}

\begin{tabular}{|c|c|c|c|c|}
\hline$\alpha[\%]$ & $I$ & $\mu_{\mathrm{I}}[\mathrm{nm}]$ & $Q_{\mathrm{I}}[\mathrm{b}]$ & IS $\left({ }^{x} \mathrm{Hg}-{ }^{204} \mathrm{Hg}\right)$ \\
\hline - & $\cdots$ & - & - & - \\
\hline$\approx-4$ & $1 / 2$ & $0.499(2)$ & - & $20.8(6)$ \\
\hline-7 & $1 / 2$ & $0.513(9)$ & - & $18.9(8)$ \\
\hline-10 & $1 / 2$ & $0.499(4)$ & - & $19.2(4)$ \\
\hline 3 & $3 / 2$ & $-0.580(6)$ & $-0.3(1.1)\left(^{*}\right)$ & $37.1(6)$ \\
\hline$\approx$ & $3 / 2$ & $-0.596(6)$ & $\begin{array}{l}\text { not jet evalua- } \\
\text { ted }\end{array}$ & $34.6(5)$ \\
\hline
\end{tabular}
$\approx 2$ not jet evaluated
$\approx 30$

$+20 \geqslant \alpha \geqslant-20(* *)$ not measured since known already

$+20 \geqslant x \geqslant-20(* *)$ from reference [16]

$\begin{array}{ccccc}32 & 13 / 2 & -0.99862(* * *) & 2.0(1.3) & 12.3(4) \\ 10 & 1 / 2 & 0.5911(5) & - & -1.7(5) \\ - & 1 / 2 & 0.4979 & - & 14.67 \\ - & 3 / 2 & -0.5513 & 0.5 & 8.91\end{array}$

Results obtained at the ISOLDE facility. The data of two stable isotopes are added for comparison; $\alpha$ is the measured $\beta$-asymmetry or $\gamma$-anisotropy corrected for background.

(*) Preliminary value.

(**) Depending of individual $\gamma$-transition.

$(* * *)$ From ref. [24].

linear increase in the optical transition frequency (this means in electron binding energy) due to a steady contraction of nuclear charge distribution. Since in $\mathrm{Hg}$ only two protons are lacking to close the shell $Z=82$, one expects these nuclei to be spherical. Hence the first term in formula (3) should be responsible for the IS

$$
\begin{aligned}
<r^{2}> & =\left\langle r^{2}>_{\mathrm{s}}+<r^{2}>_{\mathrm{s}} \frac{2}{5}<\beta^{2}>\right. \\
\delta<r^{2}> & =\delta<r^{2}>_{\mathrm{s}}+\left\langle r^{2}>_{\mathrm{s}} \cdot \frac{2}{5} \delta<\beta^{2}>\propto\right. \\
\mathrm{IS}_{\mathrm{cxp}} & =k C_{\mathrm{th}}\left[\frac{C_{\text {expr }}^{\text {spher }}}{C_{\mathrm{th}}} \delta N+0.6 A \delta<\beta^{2}>\right] .
\end{aligned}
$$

(In $(3 a)$ the mean squared radius $\left\langle r^{2}\right\rangle$ of a deformed nucleus is decomposed in a term $\left\langle r^{2}\right\rangle_{\text {s }}$ which would stem from a spherical nucleus with the same charge and volume, and a term describing the increase of $\left\langle r^{2}\right\rangle$ due to deformation at constant volume. $(3 b)$ is the corresponding change in $\left\langle r^{2}\right\rangle$ to first order and is proportional to $(3 c)$, the experimentally observed IS. $C_{\mathrm{th}}$ is the theoretical IS constant calculated on the basis of a homogenously charged nucleus expanding according to

$$
\left.R_{0}^{2}=\frac{5}{3}<r^{2}\right\rangle_{\mathrm{s}}=1.2 \mathrm{f} \cdot A^{1 / 3}
$$

However, the slope in figure 8 is too flat. As compared to theory the experimental IS is a factor of two smaller $\left(\frac{C_{\mathrm{cxp}}^{\text {spher }}}{C_{\mathrm{th}}}=0.5\right)$. This result is observed as an overall effect in all mass regions and is called IS-discrepancy. Since muonic IS reproduces it also, we should not assume the calculations of IS-constants
$C_{\mathrm{th}}$ to be wrong, but the assumptions about the change of $\left\langle r^{2}\right\rangle$ with neutron number. Obviously the charge distribution does not contract enough when neutrons are stripped. Among the theoretical suggestions about this effect we will mention the one assuming that the surface thickness of the Fermi distribution of the protons increases with decreasing $\mathrm{N}$ discussed eg. in reference [18]. This would counterbalance the shrinking of the nuclear core.

Other effects to be seen in the diagram for the region ${ }^{192} \mathrm{Hg}$ to ${ }^{205} \mathrm{Hg}$ are : (i) odd-even staggering, that is, the odd isotopes ly always closer to their lighter even neighbour. (ii) The four isomer shifts from ${ }^{193} \mathrm{Hg}^{\mathrm{m}}$ to ${ }^{199} \mathrm{Hg}^{\mathrm{m}}$. We will not go into the discussion of these effects, especially, since the full experimental accuracy as good as $1 / 100$ of the dot size for some points, can not be seen.

4.2 The Phase transition IN VLRY Light $\mathrm{Hg}$ ISOTOPES. - We would like to draw our attention finally to the large, very sharp jump in IS observed between ${ }^{181,183,185} \mathrm{Hg}$ and ${ }^{187} \mathrm{Hg}$. Suddenly $\left\langle r^{2}\right\rangle$ expands to the same value as observed for ${ }^{196} \mathrm{Hg}$ containing over $10 \%$ more neutrons. An effect of this size and sharpness can be explained only by a global, collective change in the proton distribution. We might call it therefore a phase transition of the nucleus.

What kind of phase transition ? One possibility could be the sudden appearence of strong deformation with $\beta \approx 0.3$, a great surprise so close to the magic proton number $Z=82$. Theoretical calculations of the Strutinsky type are being performed with a clear tendency to reproduce the result [19], [20] (*). Quan-

$\left({ }^{*}\right)$ Note added in proof : Hartree Fock calculations are presented at this congress by $P$. Quentin which again support this interpretation [25]. 
titative agreement is still lacking. The measurement of a quadrupole moment would be decisive, but unfortunately the nuclear spins are $1 / 2$. A search for rotational bands was negativ [21]. The theoretical work suggests a transition from a small oblate to a large prolate deformation for which also the pattern of $\mathrm{O}^{+}$energy levels in the lighter neighbours $\mathrm{Pt}$, Os give a hint [22]. Anyhow the situation differs from the one met at the onset of deformed rare earth nuclei. Large zero point motions of the nuclear shape have to be expected, since the potential minima are very flat. They would enter into the IS as well, since the deformation parameter enters into (3) by its mean squared value.

A second possible explanation is based on Wongs hypothesis of "bubble nuclei" with a hole in the centre [21], [23]. A homogeneously charged bubble with a sharp surface at the inner radius $R_{2}$ and outer radius $R_{1}$ would have approximately the mean squared radius

$$
<r^{2}>_{\mathrm{B}}=\left\langle r^{2}>_{\mathrm{s}}\left[1+\frac{5}{3}\left(\frac{R_{2}}{R_{1}}\right)^{3}\right]\right.
$$

where $\left\langle r^{2}\right\rangle_{\mathrm{s}}$ stands for the mean squared radius of a solid sphere with same volume and charge density. Assuming ${ }^{187} \mathrm{Hg}$ to be a solid sphere and the lighter ones to be bubbles, the observed jump in IS would be fitted by a ratio $\left(R_{2} / R_{1}\right) \approx 0.2$, just the value given by Wong for ${ }^{174} \mathrm{Yb}$, having the same neutron number as ${ }^{184} \mathrm{Hg}$. In the bubble model ${ }^{184} \mathrm{Hg}$ would be a double magic nucleus in the sense that the bubble state reaches its minimum energy as a function of proton and neutron number. There is still no confidence, however, whether this could be the absolute minimum as compared to the normal state and hence represent the ground state.

\section{References}

[1] Bonn, J., Huber, G., Kluge, J., Köpf, U., Kugler, L. and Otten, E. W., Phys. Lett. 36B (1971) 41.

[2] Bonn, J., Huber, G., Kluge, H.-J., Kugler, L. and OTten, E. W., Phys. Lett. 38B (1972) 308.

[3] Bonn, J., Huber, G., Kluge, H.-J., KöpF, U., Kugler, L. OTteN, E. W. and Rodriguez, J., J. Phys. Soc. Jap. 34 Suppl. (1973) 317.

[4] Kopfermann, H., Nuclear Moments (Academic Press, New York and London) 1958.

[5] Nuclear Data Tables, Section A, Vol. 5, Nr. 5-6, ed. Fuller G. H. and Cohen V. W. (Academic Press, New York and London) 1969.

[6] KJelberg, A. and Rudstam, G., The ISOLDE Collaboration, CERN Yellow Report 70-3, CERN, Geneva (1970).

[7] Lindgren, I., Proposal to the ISOLDE Committee Meeting (1972), unpublished.

[8] Otten, E. W., in : " Proc. of the Int. Conf. on the Properties of Nuclei far from the Region of B-Stability ", Leysin (1970), CERN Yellow Report 70-30, Vol. 1, p. 361, CERN Geneva (1970).

[9] Cohen Tannoudi, C. and Kasti.fr A., in : Progress in Optics, Vol. 5, p. 1 ; ed. E. Wolf (North Holland Publ. Comp., Amsterdam) 1966.

[10] Happer, W., Rev. Mod. Phys. 44 (1972) 169.

[11] Köpf, U., Besch, H. J., Otten, E. W. and von Platen, Ch., Z. Phys. 226 (1969) 297.

[12] OTteN, E. W., in : « Hyperfine Interactions in Excited Nuclei ", p. 363 ; ed. Goldring G. and Kalish R. (Gordon and Breach Science Publ., New York-LondonParis) 1971.

[13] Bonn, J., Otten, E. W., von Platen, Ch., Linder, W. MülleR, H. and SChweickerT, H., in : « Proc. of the Int. Conf, on the Properties of Nuclei far from the Region of $\beta$-Stability ", Leysin (1970), CERN Yellow Report 70-30, Vol. 1, p. 383, CERN, Geneva (1970).

[14] Schweickert, H., Dietrich, J., Neugart, R. and Otten E. W., J. Phys. Soc. Jap. 44 Suppl. (1973) 164.

[15] Capeller, U. and Mazurkewitz, M., priv. comm. and to be publ., in : Journ. of Magnetic Rresonance.

[16] Tomlinson, W. J. and Stroke, H. H., Nucl. Phys. 60 (1964) 614.

Moskowitz, P. A., Liu C. H., Fulop, G. and Stroke H. H., Phys. Rev. C 4 (1971) 620 and references cited therein.

Goorvitch, D., Davis, S. P. and Kleiman, H., Phys. Rev. 188 (1969) 1897.

[17] Laulainen, N. S. and McDermort, M. N., Phys, Lett. 39A (1969) 103.

[18] Wu, C. S., in : "Physics of the One and Two Electron Spectra, p. $429 \mathrm{ff}$; ed. Bopp F. and Kleinpoppen H., (North Holland Publ. Comp., Amsterdam), 1969.

[19] Faessler, A., Götz, U., Slalov, B., and Ledergerber, T., Phys. Lett. 39B (1972) 579.

[20] Dickmann, F. and Dietrich, K., priv. comm. and to be submitted to $Z$. Phys.

[21] Hornshøj, P., Hansen, P. G., Jonson, B., Lindahl, A., NiELSEN, O. B. and the ISOLDE collaboration, Phys. Lett. 43B (1973) 377.

[22] Finger, M., Foucher, R., Husson, J. P., Jastrebski, J., Johnson, A., Astner, G., Erdal, B. R., KJelberg, A., Patzelt, P., Hoglund, A., Malmskog, S. G. and Henck, R., Nucl. Phys. A 188 (1972) 369.

[23] Wong, C. Y., Phys. Lett. 41B (1972) 451.

[24] Reimann, R. J., Geflhood, B. D., and McDermott, M. N., Bull. Am. Phys. Soc. 16 (1971) 848.

[25] Que.vTIN, P., this volume, p. 101. 